\title{
LA CATEGORÍA SOCIAL DE VOLUNTARIADO Y SU REALIDAD EN ESPAÑA
}

\author{
JUAN SEBASTIÁN FERNÁNDEZ PRADOS \\ Universidad de Almería
}

\section{PALABRAS CLAVE ADICIONALES}

Voluntarios, Entidades de voluntariado, Tercer

sector.

\section{ADDITIONAL KEYWORDS}

Volunteers, Voluntary Organisations, Third

Sector.

RESUMEN. Los dos principales objetivos tratados en este estudio son, por una parte, construir un modelo conceptual de la categoria social de voluntariado y, por otra, cuantificar la realidad del voluntariado en España. Para ambos fines, se hace un repaso a la bibliografia sobre definiciones e investigaciones que hayan tenido como tema las entidades de voluntarios. Finalmente, el presente trabajo concluye, en primer lugar, la necesidad de aplicar criterios más rigurosos a la hora de medir el voluntariado en España desde un concepto bien delimitado y, en segundo lugar, el escaso peso que tiene el voluntariado en la sociedad española actual, a pesar del aumento de las organizaciones del Tercer Sector.

ABSTRACT. The two primary objectives of this paper are, first, to develop a conceptual model of the social category of voluntarism and, second, to quantify the actual degree of voluntarism in Spain. To these ends, bibliographic review of the definitions and research on voluntary organization is made. Finally, the paper concludes, in the first place, with a call to apply more rigorous criteria when measuring the degree of voluntarism in Spain and, in the second instance, with the observation that de gree of voluntarism in Spain and, in the second instance, with the observation that despite the increase in Third Sector organizations, voluntarism in Spanish society remains at a low level.

E-mail: jsprados@ual.es

Revista Internacional de Sociología (RIS)

Tercera Época, nº 32, Mayo-Agosto, 2002, pp. 181-198. 
RIS

REVISTA INTERNACIONAL DE SOCIOLOGIA

N 32, Mayo-Agosto, 2002

JUAN SEBASTIÁN FERNÁNDEZ PRADOS

\section{INTRODUCCIÓN}

El voluntariado se ha convertido en una realidad social que ha experimentado una gran popularidad en España, a raíz del aumento de entidades sin ánimo de lucro en las dos últimas décadas. En cualquier caso, ha sido el auge y la primacía de la sociedad civil la que ha vuelto a poner sobre el tapete la participación y protagonismo ciudadano en forma de acciones voluntarias, al amparo de las nuevos movimientos sociales englobados en el acrónimo de ONG (Pérez Díaz, 1987, 1994).

También las instituciones públicas han alentado y favorecido su difusión entre la opinión pública a través de leyes, planes y agencias especializadas; incluso las Naciones Unidas, tras constituir el día internacional del voluntariado el 5 de diciembre, declararon el 2001 como año internacional del voluntariado ${ }^{1}$. Ha sido el desarrollo legislativo desde la promulgación de la Ley 6/1996, de 15 de enero, lo que ha reforzado un proceso de institucionalización del voluntariado, construyendo, simultáneamente, una concepción determinada desde las Administraciones Públicas (Madrid, 2001).

A pesar de no ser una realidad nueva en el panorama de la acción, intervención o participación social, el término voluntariado ha provocado que cada vez más experiencias se reflejen en ella, suplantando en muchos casos, otros conceptos hasta ahora empleados, como pueden ser el de colaborador, militante, etc. Las consecuencias directas de este fenómeno apuntan, por una parte, al peso específico como categoría social día a día más utilizada y, por otra parte, una falta de definición clara y precisa del concepto teórico de voluntario (Zubero, 2001). Sin este último requisito la labor del investigador en ciencias sociales se vuelve imprecisa; o dicho de otro modo, sin una delimitación fundamentada teóricamente de la categoría de voluntariado, los resultados de los estudios e investigaciones no sólo serán incomparables, sino incontrastables con la realidad, que no se descubrirá o explicará ajustadamente.

De este modo, se pretende en este artículo presentar y revisar las principales definiciones de voluntariado para construir, posteriormente, un modelo delimitado y confrontado con términos afines de voluntariado. El repaso de las diferentes propuestas de definición, desde los diccionarios hasta las de sociólogos españoles y

\footnotetext{
' De este modo, la importancia del voluntariado ya fue reconocida por la Asamblea General de las Naciones Unidas que, en sesión de 17 de diciembre de 1985, proclamó el día 5 de diciembre de cada año como Día Internacional de las Personas Voluntarias para el Desarrollo Económico y Social. Resolución 52/17, de 20 de noviembre de 1997, en la que proclamó el año 2001 Año Internacional de los Voluntarios.
} 
extranjeros, pasando por las contenidas en la legislación, nos aportará un conjunto de notas y características que se hacen urgente antes de valorar someramente los estudios más recientes llevados a cabo en España sobre el voluntariado

\section{DEFINICIONES DE VOLUNTARIADO}

El número de definiciones de voluntariado o voluntario comienza a ser tan grande como la bibliografia que crece en los anaqueles de las bibliotecas. La repetición, las citas de otros autores precedentes, y las autorreferencias de otros muchos, nos hace pensar que en el concepto de voluntariado está apareciendo un cierto acuerdo sobre qué es el voluntariado y quién es voluntario, sobre la base de unas características comunes.

Obviamente, la primera diferencia que tendríamos que observar sería la particularidad de voluntariado frente a voluntario; el primero se refiere al conjunto de voluntarios o a la acción de éstos, mientras el segundo posee como referente al propio sujeto de la acción. Pero a pesar de este primer acuerdo y de las esperanzadoras perspectivas de unificación de criterios, la realidad nos sigue mostrando una gran diversidad de determinaciones y concreciones de estos términos.

A continuación, mostramos una docena de ejemplos que se pueden agrupar en tres grandes fuentes: la literatura terminológica, legislativa y sociológica. A la primera categoría corresponden la definición A; a la segunda la $\mathrm{B}$ y la $\mathrm{C} ; \mathrm{y}$, por último, las aportaciones de orden sociológico se ejemplifican con las definiciones D y E:

A) "voluntariado. m. Alistamiento voluntario para el servicio militar. $\| 2$. Conjunto de los soldados voluntarios. || 3 . Conjunto de las personas que se ofrecen voluntarias para realizar algo.

voluntario, ria. (Del lat. voluntarius). adj. Dicho de un acto: Que nace de la voluntad, y no por fuerza o necesidad extrañas a aquella. $\| 2$. Que se hace por espontánea voluntad y no por obligación o deber. || 3. Que obra por capricho. || 4. m. yf. Persona que, entre varias obligadas por turno o designación a ejecutar algún trabajo o servicio, se presta a hacerlo por propia voluntad, sin esperar a que le toque su vez. || 5. m. soldado voluntario." (RAE, 2001)2.

\footnotetext{
${ }^{2}$ Otro ejemplo sería: "Voluntariado. Alistamiento voluntario para el servicio militar Voluntario 1. Dictado por la voluntad: 'Acto voluntario' 2. Hecho por propia voluntad y no por obligación o por fuerza. 3. (n). Persona que se presta voluntariamente a realizar un acto. Particularmente, hombre que se presenta a realizar el servicio militar sin estar obligado a ello o antes de ser llamado" (Moliner, 1990).
} 
RIS

REVISTA INTERNACIONAL DE SOCIOLOCIA

N 32, Mayo-Agosto, 2002

JUAN SEBASTIÁN FERNÁNDEZ PRADOS

B) “A. Preámbulo [...] El voluntariado: -es una decisión voluntaria que se apoya sobre motivaciones y opciones personales; - es una forma de participación activa del ciudadano en la vida de las comunidades; -se manifiesta, generalmente, en una acción y en un movimiento organizado en el seno de una asociación; - contribuye a la mejora de la calidad de vida y a crear un mundo más solidario; - responde a los principales desafios de una sociedad que busca lograr un mundo más justo y pacifico; contribuye al fortalecimiento de un desarrollo social yeconómico más equilibrado, incluso a la creación de nuevos empleos y profesiones" ${ }^{3}$.

C) "Los términos voluntario y voluntariado nombran un trabajo emprendido por alguien por su propia cuenta o por otro fuera de su familia, sin obtener un salario directo, ejercido libremente, sin intervención del Estado ni de los organismos que de él dependen.

La acción voluntaria en beneficio de la sociedad se define como:

- La expresión de un sentimiento humanitario;

- Un medio a disposición del individuo para contribuir personalmente a la vida de la comunidad y desempeñar en ella un papel;

- Un medio para mejorar la calidad de vida para el conjunto de la sociedad;

- Un medio para la sociedad de desempeñar sus obligaciones a juicio de sus miembros;

- Un medio para expresar los intereses de los individuos y grupos" 4.

D) "El voluntariado es el que además de sus propios deberes profesionales $y$ de estatus, de modo continuo, desinteresado y responsable dedica parte de su tiempo a actividades no en favor de si mismo ni de los asociados (a diferencia del asociacionismo), sino en favor de los demás o de intereses sociales colectivos,

\footnotetext{
${ }^{3}$ Declaración Universal sobre el Voluntariado, París, 14 de septiembre de 1990; Cfr. Lambistos Roca (1996: 26).

${ }^{4}$ The Volunteer Centre Propuesta de código ético del voluntariado; (Cfr. Domingo Moratalla, 1997: 305). Un par de ejemplos más desde la perspectiva de los códigos, cartas y leyes serían los recogidos por la Carta Europea para los Voluntarios: "El trabajo voluntario puede definirse de diferentes formas pero estas definiciones deben incluir, al menos, los siguientes elementos: - Las actividades de interés económicos personal. - Carece de interés económico personal. - Se desarrolla en un marco más o menos organizado. - Es una elección libre, y se expresa por medios pacificos"; y la Ley Estatal del Voluntariado (6/1996): "Artículo 3. Concepto de voluntariado. 1. A los efectos de la presente Ley, se entiende por voluntariado el conjunto de actividades de interés general, desarrolladas por personas fisicas, siempre que las mismas no se realicen en virtud de una relación laboral, funcionarial, mercantil o cualquier otra retribuida y reúna los siguientes requisitos: a) Que tengan carácter altruista y solidario. b) Que su realización sea libre, sin que tengan su causa en una obligación personal o deber juridico. c) Que se lleven a cabo sin contraprestación económica, sin perjuicio del derecho al reembolso de los gastos que el desempeño de la actividad voluntaria ocasione. d) Que se desarrollen a través de organizaciones privadas o públicas y con arreglo a programas o proyectos concretos".
} 
según un proyecto que no se agota en la intervención misma (a diferencia de la beneficencia), sino que tiende a erradicar o modificar las causas de la necesidad o marginación" (Tavazza, 1995: 30).

E) "El voluntariado social vendría definido por aquella serie de personas que, voluntaria y solidariamente, deciden prestar una parte de su tiempo y de sus facultades en beneficio de otros ciudadanos que lo necesitan, en organizaciones y programas de acción social y sin recibir las contraprestaciones habituales en el mercado" (Gutiérrez Resa, 1997: 17).

En los diccionarios y enciclopedias, a veces poco actualizados, el término voluntariado y voluntario arrastra acepciones y connotaciones próximas a la milicia, ya que el nombre utilizado para aquellos que se alistaban motu proprio era el de voluntarios.

Este significado está muy arraigado en todos los países y tiene su expansión en el período que va entre las dos grandes guerras mundiales; por ejemplo, las tropas que se sumaban a las Brigadas Internacionales en España, provenientes de países europeos y de Norteamérica se les denominaba como voluntarios (Eby, 1974: 17). La tendencia de esta fuente bibliográfica, que son los diccionarios y enciclopedias, es recoger progresivamente otros sentidos más actuales cercanos a la acepción de cual se hace más uso hoy día y que se está tratando en el presente trabajo.

La segunda fuente empleada, que son las convenciones y normas reflejadas en las leyes de todos los ámbitos, nos aporta una visión muy particular, pero efectiva a la vez. Las disposiciones legales poseen la virtualidad de estar presentes en los distintos niveles donde los estamentos gubernamentales ostentan influencia, desde el ámbito internacional, nacional e incluso regional.

Además, tienen la particularidad de recoger las demandas sociales para la regulación y determinación de un fenómeno social como es el voluntariado, enmar-

\footnotetext{
${ }^{5}$ Existen otras definiciones recogidas por otros sociólogos o tratados sociológicos como son: "El voluntariado social acaba entendiéndose como un servicio gratuito y desinteresado que nace de la triple conquista de la ciudadanía; como un ejercicio de la autonomía individual, de la participación social y de la solidaridad para con los últimos" (García Roca, 1994: 62); "Voluntariado es el ciudadano que libremente, no en ejecución de obligaciones morales específicas o deberes jurídicos, inspira su vida - en público y en privado- en fines de solidaridad. Por lo tanto, cumplidos sus deberes civiles y de estado, se pone desinteresadamente a disposición de la comunidad, promoviendo una respuesta creativa a las necesidades emergentes de su territorio, con atención prioritaria a los pobres, los marginados, los impedidos. Dedica energías, capacidades, tiempo y los medios de que eventualmente disponga en iniciativas para compartir, realizadas especialmente a través de acciones de grupo. Iniciativas abiertas a una leal colaboración con las instituciones públicas y las fuerzas sociales, conducidas con una adecuada preparación específica; realizadas con continuidad de intervenciones, destinadas a los servicios inmediatos o la indispensable eliminación de las causas de injusticia y de toda opresión de la persona" (Nuovo Dizionario di Sociologia, Cfr. Tavazza, 1995: 31).
} 
RIS

cándolas y respaldándolas "oficialmente", y sentando las bases para su desarrollo posterior. Por último, sus consideraciones están encaminadas, fundamentalmente, a delimitar y clarificar qué es el voluntariado frente a cualquier otra actividad; lo cual conduce, definitivamente, a considerar la legislación como un referente obligado en toda definición o elaboración teórica.

A pesar de la interesante perspectiva legislativa sobre esta cuestión, no han dejado de plantearse severas dudas e interrogantes sobre el sentido de normativizar una realidad social nacida exclusivamente de la voluntad libre, espontánea y solidaria de las personas. Tavazza (1995: 34) se sitúa radicalmente en contra de la existencia de un marco legal para el voluntariado porque el Estado no tiene derecho a intervenir en este campo a no ser que se le demande.

Frente a esta postura, otros autores justifican la construcción de un marco jurídico, a partir de la recomendación de 21 de junio de 1985 del Comité de Ministros del Consejo de Europa, para la detección de obstáculos al voluntariado, el deslinde del trabajo voluntario y del asalariado, la solución de conflictos entre organizaciones y voluntarios y, en definitiva, para el conocimiento de las posibles medidas públicas de apoyo al voluntariado (Gutiérrez Resa, 1997: 305).

En conclusión, la juridificación es un proceso tan inevitable como ambivalente en su interpretación y valoración ${ }^{6}$. La realidad del voluntariado como la de los movimientos sociales va a padecer inapelablemente el empuje de la Administración y la colonización de la normatividad para fomentar y garantizar la libre participación pero también para una mayor eficacia en su control.

$\mathrm{El}$ avance ineluctable de la legislación en todos los órdenes internacionales, nacionales y regionales, junto a la constatación de su progresiva presencia y especialización que abarca desde normas genéricas sobre asociacionismo, específicas sobre el voluntariado (por ejemplo, ambiental, educativo, cultural...).

La tercera fuente de la que emanan las últimas definiciones citadas está en las ciencias sociales, fundamentalmente, ya que los autores que la redactaron son sociólogos expertos en la materia del voluntariado o en participación ciudadana. La coincidencia es importante en lo que respecta a los rasgos generales y básicos del voluntariado, como es la gratuidad y la libertad, pero a estos se añaden dos características especialmente sugerentes y ostentadoras de un fuerte componente comportamental y social.

\footnotetext{
6 "Todo induce a pensar, por tanto, que la juridización del voluntariado es un proceso inevitable. A priori, esa intervención administrativa en la organización, promoción y orientación de la acción voluntaria puede ser saludable [...] De otro lado, evidentemente, la intervención administrativa en el voluntariado (regulándose, estableciendo medidas de fomento y control, criterios de organización, etc.) y a través del voluntariado, en el conjunto de la política y la acción social, conlleva importantes riesgos" (García Inda, 1996: 202).
} 


$\begin{array}{r}\text { R I S } \\ \text { LA CATECORÍA SOCIAL DE VOLUNTARIADO Y SU REALIDAD EN ESPAÑa } \\ \hline\end{array}$

La primera idea, insistente en casi todas las delimitaciones de voluntariado, es su carácter indubitablemente altruista, generoso y solidario. Expresiones como desinteresado y responsable y servicio gratuito aparecen no sólo para reforzar la idea de la nula retribución de la actividad del voluntario sino que, también, apuntan hacia un estilo, actitud y comportamiento personal y particular basado en valores positivos y pro-sociales.

La segunda consideración reiterada en los cinco textos es la finalidad transformativa de la sociedad en orden a mejorarla o a hacerla más justa. Afirmaciones tales como "en beneficio de otros ciudadanos", "modificar las causas de la necesidad o marginación" y "eliminación de las causas de injusticia" señalan que en el trasfondo o en el fin de la acción voluntaria existe una clara intención no sólo asistencial, sino también de transformación y cambio social en una línea, obviamente, de valores como la justicia, la libertad y la igualdad.

\section{SOCIOLOGÍA DEL VOLUNTARIADO: HACIA UN MODELO CONCEPTUAL}

Tavazza llama la atención sobre las dificultades que tiene ofrecer una definición global al término voluntariado, entre otros motivos por su falta de claridad y especificidad, que lo ha convertido en un concepto amplio y genérico, vaciándolo progresivamente de significado.

La variedad de interpretaciones a lo largo de la historia, por una parte, y las innumerables dimensiones en las experiencias del voluntario, han provocado una confusión aún mayor en la idea de voluntariado; por esta razón, la opción más conveniente es hablar de voluntariados mejor que del singular voluntariado y distinguir entre el nuevo voluntariado (servicios a la comunidad, en general) el viejo (asociado al ámbito militar) o emergente voluntariado (espontáneo $\mathrm{e}$ individualista)(Tavazza, 1995: 37-38).

Estos nuevos voluntariados poseen unas características que lo definen y, sobre todo, los delimitan, de otras experiencias asociativas o actividades, con las que habitualmente se han llegado a confundir, creyendo que se hablaba de lo mismo. Iniciaremos un recorrido jalonado por tres aproximaciones: la primera y más general partirá del sentido original y etimológico de la palabra voluntario; la segunda nos delimitará el concepto de voluntariado frente a otros términos y actividades con las que hoy día se confunden, deduciéndose de su confrontación rasgos esenciales; y por último, plantearemos una tercera aproximación contraponiendo el voluntariado con otras situaciones dentro de las asociaciones con las que se puede llegar a provocar equívocos.

En primer lugar, el punto de vista histórico y etimológico nos lleva a constatar que el voluntariado surge fruto de un acto de la voluntad libre, es decir, el individuo decide por sí mismo optar por este tipo de actividad, independientemente de la motivación o el contexto social en el que viva. Este primer rasgo se opone 
RIS

a cualquier otra vivencia de instituciones primarias o básicas de vinculación ineludible, impuestas por una determinada cultura o sociedad, como es el caso de la ciudadanía o la familia que obliga a sus nacionales o a su parentesco su pertenencia obligatoria bajo contrato (derechos y deberes) sin previo consentimiento. No sólo desde la libertad se opta por ser voluntario sino también es libre la elección de la organización y de la tarea que más se adapte a las capacidades humanas y profesionales.

Pero, además, de esta primera característica (voluntad libre) que se inicia en la misma etimología de la palabra voluntario, nos encontramos con otras cuatro confrontaciones que pueden ser útiles para completar la descripción y aportar nuevos elementos a la definición del voluntariado (ver figura 1).

a) Voluntariado y profesionalismo. La diferencia crucial entre el voluntario y el profesional es que el primero realiza su actividad sin recibir nada a cambio, es decir, gratuitamente, mientras que el segundo lleva a cabo su labor, principalmente, por la compensación o gratificación económica en forma de salario. Por este motivo, las entidades voluntarias asociadas a la P.P.V.E. (Plataforma para la Promoción del Voluntariado en España) consideran que cualquier tipo de retribución en especie o pecuniaria desvirtúa o entra en contradicción con el mismo sentido del ser voluntario; comentario que aparece a raíz de la valoración realizada sobre la Ley Estatal del Voluntariado y sus propuestas de gratificación y compensación por servicios prestados.

Si las organizaciones de voluntariado, por una lado, se esfuerzan por delimitar su identidad frente a cualquier intrusismo lucrativo, las asociaciones de profesionales, por otra parte, muestran sus objeciones al auge del voluntariado por diversos motivo (informalidad, carencia de experiencia, motivaciones de mala conciencia, poca profesionalidad y formación, sustituyen puestos de trabajo, son fácilmente manipulables...). Pero a pesar de estas críticas, la convivencia y la colaboración entre estos dos tipos de actividades va a ser cada vez más frecuente, tanto en el marco de las Administraciones Públicas (por la crisis del Estado del Bienestar) como en las propias organizaciones no gubernamentales (por la institucionalización); lo cual va a exigir una mayor delimitación de las fronteras entre unos y otros y la colaboración de ayuda mutua (profesionales que solicitan voluntarios y las organizaciones de voluntariado que reclaman a profesionales para una formación más adecuada) ${ }^{7}$. Incluso Naciones Unidas ha

\footnotetext{
${ }^{7}$ En Voluntarios y Profesionales. ¿Consecuencia o cooperación?, Bernardo Corral desarrolla en un amplio cuadro cómo se ven unos y otros y la valoración positiva y negativa en distintos aspectos (Cfr. en Noguera Arrom, 1997: 21). Concretamente se plantean dos tipos de conflictos entre el trabajo remunerado y el trabajo voluntario, uno de intereses (horarios, lugar físico de trabajo...) y otros de valores (objetivos y métodos de trabajo), aunque el más importante es la imagen positiva o negativa que tenga un grupo del otro (Blanco Puga, 1996: 131-132).
} 
Figura 1.

Caracteristicas del voluntariado social frente a otras actividades

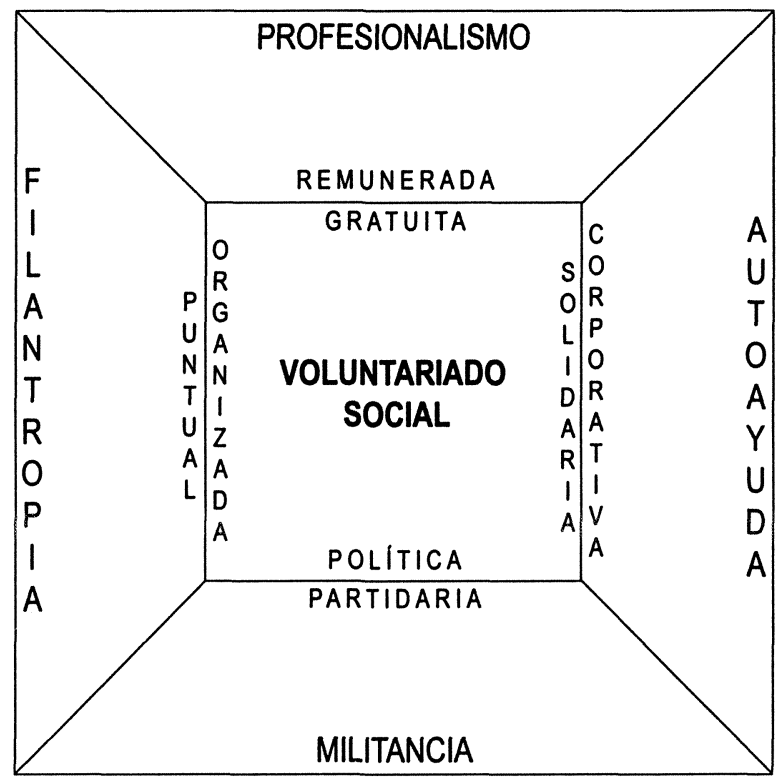

matizado este criterio al considerar que el servicio voluntario es aquel que no se lleva a cabo principalmente por lucro financiero y si el reembolso monetario que la gente recibe por el trabajo que hacen equivale al "valor de mercado" del trabajo o más, abriendo así la posibilidad de remunerar la actividad voluntaria (Dingle, 2001).

b) Voluntariado y militancia. La distinción entre actividad política, entendida en sentido etimológico de interés por la calidad de vida en la ciudad, y la actividad partidaria, definida por una tendencia ideológica excluyente y una disciplina interna férrea, nos ofrece los pilares que oponen voluntariado y militancia. Algunos autores han pretendido diferenciarlos basándose en las mediaciones propuestas por cada uno de ellos; así, aunque sea cierto que ambos, voluntariado y militancia, se presentan como respuestas frente a la pobreza, las miserias y la injusticia social, uno dirige sus esfuerzos a corregir los hechos o carencias tal y como se van manifestando, y el otro se preguntará más por las causas y sobre ellas orientará prioritariamente su acción (Rodríguez, 1997: 5) ${ }^{8}$.

\footnotetext{
${ }^{8}$ La obra de Alejandro Rodríguez hay que ubicarla dentro del Movimiento Cultural Cristiano que considera que tanto las ONG como los Voluntariados son cánceres extendidos en las tres últimas
} 
RIS

REVISTA INTERNACIONAL DE SOCDOLOGIA

No 32, Mayo-Agosto, 2002

JUAN SEBASTIÁN FERNÁNDEZ PRADOS

Esta aportación, posiblemente confunda dos tipos de voluntariado el postmoderno y el disidente, y quiera identificar este último con la militancia, término que expresa realmente la vinculación a una determinada ideología partidista. El voluntariado, como veremos en nuestra investigación, posee un amplio espectro de orientaciones que supera o permanece al margen de las escalas de posición ideológica, compartiendo básicamente una orientación axiológica y cultura política determinada, aunque los métodos (más o menos revolucionarias) o las exigencias de cambio (más o menos radicales), no sean las mismas.

c) Voluntariado y auto-ayuda. Las organizaciones y asociaciones dedicadas a favorecer a los miembros del mismo grupo, llamadas de auto-ayuda o de ayuda mutua, se han multiplicado en los últimos años, junto a estas han aparecido las entidades voluntarias sociales propiamente dichas que aglutinan a personas que libremente optan por trabajar en favor de colectivos desfavorecidos a los cuales no pertenece. Aunque, ciertamente, las primeras pueden acoger a voluntariado también, solo tiene sentido hablar en sentido restringido de él cuando responda al principio de solidaridad o de altruismo, es decir, la acción no beneficia directamente al voluntario $o$ al grupo al que pertenezca por compartir alguna característica similar'. El principio de la acción solidaria, definida como aquella que se realiza con carácter universal (frente a localistas y corporativistas), práctico (frente a teóricos y demagogos) y justa (frente a la meritócratas e igualitarios injustos). El voluntariado, en términos de García Roca, practica y representa la cultura del don de la gratuidad y de la solidaridad, que en el horizonte de la emancipación se completa con el conflicto y la marginalidad, la comunicación y la vulnerabilidad.

d) Voluntariado y filantropia. Los aspectos más destacables de la filantropía que los contrapone al voluntariado son el carácter puntual de sus acciones y la conducta individual que los lleva a efecto en la mayoría de las ocasiones. Mientras, el voluntariado cuando lleva a cabo su labor, la realiza en el doble marco de una entidad que le ofrece un respaldo colectivo y grupal, y de un proyecto más amplio que le supone una continuidad y seguimiento.

A la última aproximación le corresponde discernir el sentido distintivo de la figura del voluntario, en sentido estricto, frente a otros dos conceptos con los que

\footnotetext{
décadas en nuestra sociedad. Las primeras institucionalizan las respuestas al imperialismo reinante en nuestro mundo, a base de ponerle paños calientes y nunca aplicarle el bisturí; los segundos se presentan como el ideal de tipo humano que nunca combate las causas de los problemas (Presentación de Julián Gómez del Castillo al libro "Voluntariado y Militancia").

${ }^{9}$ Juan Ramón Calo incluye a las asociaciones de ayuda mutua como un tipo de organización de voluntarios, aunque afirme que en sentido estricto no lo son (Calo, 1995: 32). Tavazza distingue de un modo más severo e identifica a las asociaciones de ayuda mutua de corporativistas, aunque realicen sus tareas de modo gratuito, no desarrollan su función con principios de universalidad y de apertura solidaria hacia todos (Tavazza,1995: 39).
} 
se suele intercambiar y que representan más bien otros modos de participación en las organizaciones de voluntariado. El socio y el colaborador eventual son pilares sobre los cuales se sustenta, evidentemente, las entidades y ONG, pero carecen de los elementos claves detentados por el voluntariado, tal y como pretendemos construir en nuestro modelo. Conforme vayamos elaborando un mayor número de criterios, el número de personas que al final consideraremos voluntarias será menor ya que estos elementos nuevos que vamos a plantear, excluyen a un número mayor de personas.

En primer lugar, el voluntariado no coincide plenamente con la figura del socio o, dicho de otra manera, el estar simplemente afiliado, cotizando a través de una aportación fija o a través de donaciones esporádicas no es condición suficiente para contemplar a esta persona como voluntaria en el ámbito social. Esta diferenciación se ve reflejada en varios estudios que se realizan en el mundo sobre voluntariado y donaciones; como por ejemplo, los referidos anteriormente y llevados a cabo en oleadas cada dos años por la organización no lucrativa Independent Sector ${ }^{10}$ (Sector Independiente) en Estados Unidos, en la cual se distingue entre voluntarios (volunteers) y donantes (giving). Para entrar en el cómputo de los voluntarios es conditio sine qua non dedicar un tiempo en acciones voluntarias y no sólo dinero.

En segundo lugar, el voluntariado no pretende ser una acción eventual o colaboración esporádica, y de este modo los trabajos elaborados por el Instituto John Hopkins, mencionados más arriba, distinguen también entre voluntario en sentido amplio y en sentido estricto. El voluntariado en sentido amplio sería aquel que dedica una hora al mes, como mínimo, a una organización no lucrativa; mientras que el voluntario en sentido estricto se referiría a aquel que dedica más de 16 horas al mes o cuatro horas a la semana a una organización del Tercer Sector.

Después de este recorrido delimitador del concepto de voluntariado, defendemos que en el contexto de la investigación social debe aplicarse en los estudios y encuestas la versión más restrictiva de voluntariado, que englobaría las siguientes cuatro características en torno a la acción desarrollada por estas personas:

a) Acción altruista y de heteroayuda. Esta dimensión de la acción voluntaria es la que la sitúa en el ámbito de la solidaridad y la ha vinculado a ella desde distintas perspectivas denominando a las propias organizaciones como solidarias, redes de solidaridad, y a los participantes como solidarios. De este modo quedan fuera todas aquellas personas que desarrollan una acción cuyo beneficio revierta de manera directa o indirecta sobre un colectivo al que ella misma pertenece; es el caso de aquellas personas jóvenes que participan en una asociación juvenil, de los inmigrantes que colaboran en organizaciones de inmigrantes...

\footnotetext{
${ }^{10}$ Independent Sector (URL: http://www.indepsec.org).
} 
b) Acción gratuita y desinteresada. Ha sido hasta ahora una de las dimensiones más subrayadas en la legislación y en la etimología del concepto de voluntariado, al nacer del más estricto "querer" y "deseo" de la persona sin esperar nada a cambio de dinero o en especie. Este aspecto de la acción del voluntariado se encuentra en entredicho, pero cualquier cuestionamiento de ésta sería poner en tela de juicio su propia esencia, como se ha comentado anteriormente.

c) Acción organizada y programada. La acción voluntaria y las organizaciones de voluntariado deben permanecer indisolublemente unidas por ser el marco, tanto para situarla como para interpretarla y clasificarla. Sin esta ubicación la acción se reduciría, como se dijo más arriba, a un acto voluntarioso y filantrópico que responde a acciones puntuales, inconexas e independientes que carecen de la coherencia y amparo formal e institucional necesario.

d) Acción continuada y frecuente. Como resultado y en íntima relación con la anterior dimensión, la acción voluntaria estará incardinada en una organización y programa de intervención social; esta consideración hace pensar que el tiempo y la frecuencia del trabajo voluntario deben poseer unos mínimos. La pregunta sería cuál es el límite entre un mero colaborador esporádico y un voluntario en el contexto de una asociación y programa de trabajo; en este sentido, la cantidad mínima para entender como voluntariado estaría en las propuestas que están realizando diversas encuestas, en torno a 4 horas semanales aproximadamente; y en todo caso diferenciando entre voluntario en sentido amplio (al menos una hora al mes) y en sentido estricto (4 horas semanales) (Ruiz Olabuénaga, 2000; 2001).

Por último, la acción voluntaria y el voluntariado social es política en tanto que participa en la construcción de la polis a través de una opción de cuidado, atención o, dicho en palabras más próximas a este ámbito de caridad, beneficencia o solidaridad con los más desfavorecidos y débiles de la sociedad.

Esta afirmación puede resultar hasta cierto punto chocante con algunos esfuerzos de determinados autores por diferenciar entre actividad política y no política, englobando en esta última las acciones llevadas a cabo en el contexto de las organizaciones caritativas, reservando así para la participación política las acciones tales como el voto, la asistencia a mítines o la colaboración en la campaña de algún partido político (Verba, Schlozman, y Brady, 1998: 40 y ss.).

La frontera entre acción política y no política no está clara, como han reconocido los propios autores que han declarado como punto de partida operativo esta segregación. Una de las razones ocultas de esta dificultad se encuentra en el reduccionismo sufrido por el concepto de "política", restringido al ámbito de las actividades desarrolladas alrededor de un partido político o de las actividades de una campaña política. El proceso de identificación de toda actividad política por aquella realizada en el seno de o para los partidos políticos, ha generado que hacer política se iguale a elegir una determinada opción partidista. El sentido amplio de 
política, como la construcción y cuidado del todo, o "polis", para que el individuo o ciudadano alcance a su vez la felicidad aristotélica, abre las puertas para que la acción voluntaria adquiera su dimensión política".

\section{ESTUDIOS SOBRE EL VOLUNTARIADO EN ESPAÑA DURANTE LOS AÑOS NOVENTA}

Los años noventa han traído consigo un aumento espectacular de las investigaciones sobre voluntariado, mientras que en el decenio anterior escasean los ejemplos que traten de un modo científico el estudio de dicho fenómeno social. Esta constatación subraya la interrelación y el mutuo reforzamiento existente entre el aumento o protagonismo de determinada realidad social y su estudio por parte de los investigadores sociales; porque es precisamente en los años noventa cuando el número de voluntarios y de asociaciones de voluntariado social han tenido su boom más importante en nuestro país.

Las características generales de estas investigaciones han sido el predominio de aquellas que han pretendido tomar la medida cuantitativa para determinar las dimensiones, perfiles, afianzamiento institucional, y rasgos de carácter más general de los participantes en las organizaciones del Tercer Sector. Los problemas surgen cuando los conceptos varían (voluntariado en cualquier institución, voluntariado sólo en organizaciones no lucrativas, voluntariado social en el ámbito estricto del bienestar social, etc.) o cuando las fuentes cambian (Registro Nacional de Asociones, informaciones facilitadas por las organizaciones voluntarias, encuestas, etc.). En conclusión, la disparidad de los resultados es harto evidente, como ocurre en los niveles de asociacionismo, cuando los fundamentos teóricos y metodológicos no están sometidos a los mismos criterios (tabla 2).

Otro rasgo común a la mayoría de las investigaciones sobre el voluntariado, ha sido la escasa presencia de la dimensión política implicada o derivada de la

\footnotetext{
"Enrique Falcón propone una retahíla de rasgos que posee o debería poseer el voluntariado social en su dimensión política; este conjunto de objetivos no deja de ser en el fondo una relación de pretensiones y deseos dirigida hacia los mismo voluntarios: abrir e interpelar procesos personales (rompiendo complicidades); hacer visible una cultura de la subversión; narrar lo invisible; recrear los frentes comunes; restituir el protagonismo de la voz del otro; potenciar su interlocución institucional; desarrollar estrategias creativas de intervención política; incidir sobre el desarrollo del tejido comunitario del entorno; desnormativizarse; rigor teórico (Falcón, 1997: 14-24).
} 
RIS

REVISTA INTERNACIONAL DE SOCIOLOCIA

No 32, Mayo-Agosto, 2002

JUAN SEBASTIÁN FERNÁNDEZ PRADOS

acción colectiva llevada a cabo por los voluntarios ${ }^{13}$. Los contenidos de los análisis han estado encaminados a relacionar el voluntariado con las motivaciones individuales, con las relaciones entre las mismas organizaciones y con otras instituciones sociales, con la movilización de recursos económicos y la cuantificación de los participantes, con la imagen y su valoración por parte de la opinión pública, etc.

El predominio de investigaciones mediante técnicas cuantitativas responde en gran medida al protagonismo que posee el uso de dichas técnicas entre los mismos científicos sociales. A esta razón general y aplicable a cualquier otro campo de estudio se une otra explicación que está asociada al momento inicial en el desarrollo de las investigaciones dedicadas al voluntariado; esto significa que en un primer momento el empeño básico de los autores es poder medir y cuantificar las dimensiones del fenómeno social de las organizaciones sollidarias o voluntarias ${ }^{14}$.

Tabla 2.

Número de voluntarios en España según los últimos estudios

\begin{tabular}{lrr}
\hline Fuentes $^{12}$ & Año & Voluntarios \\
\hline Johns Hopkins University & 1995 & 253.600 \\
Plataforma para la Promoción del Voluntariado en España & 1996 & 286.403 \\
Fundación BBVA & 1998 & (amplio) 2.931 .219 \\
& & (estricto) 1.026 .482 \\
Fundación Tomillo & 1999 & 1.073 .636 \\
CIS & 2001 & 1.800 .000 \\
\hline
\end{tabular}

${ }^{12}$ Johns Hopkins University (Salamon y Sokolowski, 2001), Plataforma para la Promoción del Voluntariado en España (Cortes, Hernán y López, 1998); Fundación BBVA, extrapolación a partir de un estudio de CINDES (Ruiz Olabuénaga, 2000), Fundación Tomillo (Fundación Tomillo, 2000); CIS, calculado a partir de 5,7\% de la población mayor de 18 años en España (Estudio 2419).

${ }^{13}$ Morán reconoce que a partir de los años noventa, de la crisis evidente de la política convencional representada por los partidos políticos y la aparición de las ONG, se ha comenzado a abrir una puerta de relación entre acción colectiva y cultura política: "Los significativos cambios en la participación y la acción colectiva que han tenido lugar en España a lo largo de la década de los noventa, concretamente el renacimiento de nuevos y viejos movimiento sociales y el auge de las ONG y de otras formas de voluntariado, han provocado que se establezca una vía de comunicación entre los estudios de cultura política y las investigaciones sobre la acción colectiva" (Morán, 1999: 117).

${ }^{14} \mathrm{Sin}$ llegar a ser exhaustivos se podría señalar, por un lado, algunos estudios cualitativos sobre entidades de voluntariado en España (Funes, 1995; Rodríguez Cabrero y Monserrat, 1996) y otras investigaciones fundamentalmente cuantitativas sobre voluntariado, sector no lucrativo o Tercer Sector, ampliamente mayoritarias (Cortés, Hernán y López, 1998; Cabra de Luna, 1998; Ruiz de Olabuénaga, 1998; Martínez Sánchez, 1998; Analistas Financieros Internacionales, 1999, etc.) 
Por último, cabe resaltar como las investigaciones más importantes realizadas en España en los últimos diez años; enfatizan un elemento común al mostrar o intuir la vinculación del fenómeno del voluntariado y sus organizaciones a un Tercer Sector. Esta afirmación o intuición de pertenecer a un sector no lucrativo se hace explícito cuando el propósito de partida es determinar algún aspecto de dicho ámbito, como aparece en los títulos de algunas investigaciones, o también en aquellas otras que parten de casos únicos como la ONCE, Cáritas, Cruz Roja o Amnistía Internacional; en un caso y en otro, la conclusión se encamina a ligarlas con el Tercer Sector y su cultura solidaria (Ruiz de Olabuénaga, 2000; Analistas Financieros Internacionales, 1999).

Pero más allá de los rasgos que comparten las investigaciones y estudios sobre el voluntariado en España, existe otro punto de desencuentro desde la propia opinión pública. Un grupo importante de personas que colaboran con su tiempo de manera desinteresada en las ONG, y que podríamos denominar como voluntarios, manifiestan, simultáneamente, no pertenecer a ninguna de estas organizaciones. En el estudio del CIS del año 2001, un 5,7\% declara dedicar parte de su tiempo y de manera desinteresada a las ONG, cuando en una pregunta anterior sólo un 4\% afirmaba pertenecer a estas entidades, lo cual resulta una diferencia importante (Tablas 3a. 3b.). Esto nos lleva a pensar que también la opinión pública posee

Tabla 3a.

Personas que dedican tiempo y pertenecen a ONG (\% columna)

\begin{tabular}{l|l|c|r|r}
\hline & & \multicolumn{2}{|c|}{ Dedica tiempo a las ONG } & Total \\
& & Si & No & \\
\hline \multirow{3}{*}{ Está vinculado a ONG } & Pertenece & $35 \%$ & $3 \%$ & $4 \%$ \\
& Ha pertenecido & $7 \%$ & $1 \%$ & $2 \%$ \\
Total & Nunca ha pertenecido & $58 \%$ & $96 \%$ & $94 \%$ \\
& & $100 \%$ & $100 \%$ & $100 \%$ \\
\hline
\end{tabular}

Fuente: Elaboración propia a partir de CIS 2419.

Tabla 3b.

Personas que dedican tiempo y pertenecen a ONG (\% fila)

\begin{tabular}{l|l|cc|c}
\hline & & \multicolumn{2}{|c|}{$\begin{array}{c}\text { Dedica tiempo a las ONG } \\
\text { Si }\end{array}$} & Total \\
& & Sertenece & $55 \%$ & $100 \%$ \\
Está vinculado a ONG & Ha pertenecido & $26 \%$ & $74 \%$ & $100 \%$ \\
& Nunca ha pertenecido & $4 \%$ & $96 \%$ & $100 \%$ \\
Total & & $6 \%$ & $94 \%$ & $100 \%$ \\
\hline
\end{tabular}

Fuente: Elaboración propia a partir de CIS 2419. 
RIS

REVISTA INTERNACIONAL DE SOCIOLOCIA

№ 32, Mayo-Agosto, 2002

una noción equívoca de voluntariado o al menos distinta de una concepción más rigurosa que implica, entre otros criterios, que la persona voluntaria pertenezca a la entidad no lucrativa.

En resumen, y según el barómetro del CIS de mayo de 2001, sólo había un dos por ciento de la población española que perteneciera a una ONG y dedicara parte de su tiempo, de manera desinteresada, a la labor desarrollada por ella. Este dato contrasta, fuertemente, con los resultados de algunas investigaciones, con el incremento de las entidades sin ánimo de lucro y, sobre todo, con la situación de otros países con más tradición de voluntariado, como es el caso de EE. UU. ${ }^{15}$

\section{CONCLUSIONES}

A pesar de que la existencia de las organizaciones caritativas y de las personas que han colaborado en ellas, posee una larga tradición de siglos en el Estado español; los conceptos de voluntario, ONG, entidades de voluntariado, etc. en el contexto de la acción social y de la participación pública de la sociedad civil, son relativamente recientes en el acervo léxico del uso cotidiano y del habla.

Esta novedad se ha visto acompañada de un crecimiento importante en las dos últimas décadas en España de organizaciones no lucrativas y de voluntarios, lo que ha motivado, simultáneamente, un aumento en el número de investigaciones y estudios, sobre todo a finales de los noventa. De este modo, nos encontramos frente a una nueva categoría social, el voluntariado, que está suponiendo no sólo una parcela cada vez más importante de la realidad social, sino un reto para las ciencias sociales, tanto en su dimensión cuantitativa como cualitativa.

Los resultados de estos trabajos evidencian la necesidad de revisar y delimitar la categoría social de voluntariado para un adecuado estudio, ya que la disparidad de los datos obtenidos es una consecuencia directa, en muchos casos, de las diferencias de criterios metodológicos y de las distintas conceptualizaciones del objeto de investigación.

Así, algunas de las características ineludibles que se han tomado en consideración en el presente trabajo, y que ayudan a desarrollar un concepto de voluntariado más riguroso son: acción libre, sin interés económico o gratuidad, de ayuda a otras personas, en el contexto de una asociación, cierta continuidad y tiempo. Estos y otros criterios ofrecen la posibilidad de construir instrumentos más precisos a la hora de cuantificar y, en definitiva, de conocer la realidad del voluntariado en España.

${ }^{15}$ La organización Independent Sector llevó a cabo una encuesta telefónica a un total de 4.216 estadounidenses mayores de 21 años sobre donaciones y actividades voluntarias entre mayo y julio de 2001. Los resultados fueron los siguientes: el $89 \%$ realizó alguna donación y el $44 \%$ desarrolló actividades de voluntariado en los doce meses previos a las entrevista (Independent Sector, 2001). 


\section{REFERENCIAS}

ANALISTAS FINANCIEROS INTERNACIONALES (1999), El Tercer Sector: El Voluntariado en la Comunidad de Madrid, Madrid, Instituto de Estadística de la Comunidad de Madrid.

BLANCO PUGA, $M^{\mathrm{a}}$.R. (1996), "Trabajadores voluntarios-trabajadores remunerados: Reflexión sobre unas relaciones que tienen que ser posibles", Documentación Social, ${ }^{\circ} 104$, pp. 129141 .

CABRA DE LUNA, M.A. (1998), Las fundaciones como parte integrante del Tercer Sector en España, Tesis doctoral, Granada, Universidad de Granada,.

CALO, J.R. (1995), ¿Qué es ser voluntario?, Madrid, Plataforma para la Promoción del Voluntariado en España.

CORTES, L., M.J. HERNÁN y O. LÓPEZ (1998), Las organizaciones de voluntariado en España, Madrid, Plataforma para la Promoción del Voluntariado en España.

DINGLE, A. (2001), La medición de la acción voluntaria: una guia práctica, Washington, DC, Independent Sector.

DOMINGO MORATALLA, A. (1997), Ética y voluntariado. Una solidaridad sin fronteras, Madrid, PPC.

EBY, C. (1974), Voluntarios norteamericanos en la guerra civil española, Barcelona, Ediciones Acervo.

FALCÓN, E. (1997), Dimensiones politicas del voluntariado, Barcelona, Cristianisme i Justícia.

FUNDACIÓN TOMILLO (2000), Empleo y trabajo voluntario en las ONGs de acción social, Madrid, MTAS.

FUNES, M. J. (1995), La ilusión solidaria: las organizaciones altruistas como actores sociales en los regimenes democráticos, Madrid, UNED.

GARCÍA INDA, A. (1996), “Aspectos legales del voluntariado: el modelo de la Ley 6/1996, de 15 de enero", Documentación Social, $\mathrm{n}^{\circ}$ 104, pp. 201-236.

GARCÍA ROCA, J. (1994), Solidaridad y voluntariado, Santander, Sal Terrae.

GUTIÉRREZ RESA, A. (1997), Acción social no gubernamental. Análisis y reflexiones sobre las organizaciones voluntarias, Tirant lo blanch, Valencia.

HODGKINSON, V. y M. WEITZMAN (1996), Giving and Volunteering in 1996, URL: http: //www.indepsec.org/media/gv_summary.html.

INDEPENDENT SECTOR (2001), Giving and Volunteering in the United States 2001: Key Findings, Washington D.C, Independent Sector. 
RIS

REVISTA INTERNACIONAL DE SOCIOLOCIA

No 32, Mayo-Agosto, 2002

JUAN SEBASTIÁN FERNÁNDEZ PRADOS

LAMBISTOS ROCA, M. J. (1996), Guia del voluntariado y autoayuda, Centro de Ediciones de la Málaga, Diputación de Málaga.

MADRID, A. (2001), La institución del voluntariado, Madrid, Trotta.

MARTÍNEZ SÁNCHEZ, J. L. (1998), La imagen de las ONG de desarrollo. Para ir dimensionando el Tercer Sector, Madrid, Instituto de Estudios Políticos para América Latina y África.

MOLINER, M. (1990), Diccionario del uso del español, Madrid, Gredos.

PÉREZ-DÍAZ, V. (1987), El retorno de la sociedad civil, Madrid, Instituto de Estudios Económicos.

(1993), La primacía de la sociedad civil. El proceso de formación de la España democrática, Madrid, Alianza Editorial.

REAL ACADEMIA ESPAÑOLA (2001), Diccionario de la lengua española, Madrid, Espasa Calpe.

RODRÍGUEZ, A. (1997), Voluntariado y militancia, Madrid, MCC.

RODRÍGUEZ CABRERO, G. y J. MONSERRAT (dir.) (1996), Las entidades voluntarias en España. Institucionalización, estructura económica y desarrollo asociativo, Madrid, Ministerio de Asuntos Sociales.

RUIZ OLABUÉNAGA, J. I. (2000), El sector no lucrativo en España, Madrid, Fundación BBV.

(2001), "El voluntariado en España", Documentación Social, n 122, pp. 67-83.

SALAMON, L. y W. SOKOLOWSKI (2001), Volunteering in cross-national perspective: evidence from 24 countries, Baltimore, The Johns Hopkins University Center for Civil Society Studies.

TAVAZZA, L. (1995), El nuevo rol del voluntariado, Buenos Aires, Lumen.

VERBA. S., K.L. SCHOLZMAN, y H. BRADY (1998), Voice and Equality. Civic voluntarism in American Politics, Cambridge, Harvard University Press.

ZUBERO, I. (2001), "La sociedad civil en acción: de la institucionalización a la crítica", Inguruak boletina, $\mathrm{n}^{\circ} 77$, pp. 10-11. 